\title{
The proportion of lycopene isomers in human plasma is modulated by lycopene isomer profile in the meal but not by lycopene preparation
}

\author{
Myriam Richelle ${ }^{1 *}$, Pierre Lambelet ${ }^{1}$, Andreas Rytz ${ }^{1}$, Isabelle Tavazzi ${ }^{1}$, Anne-France Mermoud ${ }^{1}$, \\ Christine Juhel ${ }^{2}$, Patrick Borel ${ }^{3,4,5}$ and Karlheinz Bortlik ${ }^{1}$ \\ ${ }^{1}$ Nestlé Research Center, Nestec Limited, PO Box 44, CH-100O Lausanne 26, Switzerland \\ ${ }^{2}$ Avantage Nutrition, Marseille F-13012, France \\ ${ }^{3}$ INSERM, U1025 Marseille F-13385, France \\ ${ }^{4}$ INRA, UMR 1260, Marseille F-13385, France \\ ${ }^{5}$ University Aix-Marseille, Marseille F-13385, France \\ (Submitted 16 December 2010 - Final revision received 15 July 2011 - Accepted 15 July 2011 - First published online 9 September 2011)
}

\begin{abstract}
Dietary lycopene consists mostly of the (all-E) isomer. Upon absorption, (all-E) lycopene undergoes isomerisation into various ( $Z$ )-isomers. Because these isomers offer potentially better health benefits than the (all- $E$ ) isomer, the aim of the present study was to investigate if the profile of lycopene isomers in intestinal lipoproteins is affected by the profile of lycopene isomers in the meal and by the tomato preparation. Six postprandial, crossover tests were performed in healthy men. Three meals provided about $70 \%$ of the lycopene as $(Z)$-isomers, either mainly as 5-(Z) or 13-(Z), or as a mixture of $9-(Z)$ and $13-(Z)$ lycopene, while three tomato preparations provided lycopene mainly as the (all- $E$ ) isomer. Consumption of the 5 - $(Z)$ lycopene-rich meal led to a high (60\%) proportion of this isomer in TAG-rich lipoproteins (TRL), indicating a good absorption and/or a low intestinal conversion of this isomer. By contrast, consumption of meals rich in 9-( $Z$ ) and 13- $(Z)$ lycopene isomers resulted in a low level of these isomers but high amounts of the 5- $(Z)$ and (all- $E$ ) isomers in TRL. This indicates that the 9-( $Z$ ) and 13-(Z) isomers were less absorbed or were converted into 5- $(Z)$ and (all- $E$ ) isomers. Dietary $(Z)$-lycopene isomers were, therefore, differently isomerised and released in TRL during their intestinal absorption in men. Consuming the three meals rich in (all- $E$ ) lycopene resulted in similar proportions of lycopene isomers in TRL: $60 \%$ (all- $E$ ), $20 \% 5-(Z), 9 \% 13-(Z), 2 \% 9-(Z)$ and $9 \%$ unidentified $(Z)$-isomers. These results show that the tomato preparation has no impact on the lycopene isomerisation occurring during absorption in humans.
\end{abstract}

Key words: Lycopene: (Z)-isomers: Carotenoids: Isomerisation: Absorption

Lycopene is an acyclic $\mathrm{C}_{40}$ non-polar carotenoid, present in several dietary sources such as tomato, watermelon, guava and apricot. Although not being a provitamin A, lycopene has been shown to have multiple biological activities including decreasing the risk of prostate cancer ${ }^{(1)}$, inhibition of cell proliferation, migration and invasion in breast of endometrial and liver carcinoma cells ${ }^{(2-7)}$, and prevention of mutagenesis and chromosome instability ${ }^{(8,9)}$. In addition, a variety of epidemiological trials indicated that high intakes of lycopene-containing foods (primarily tomato products) or elevated blood lycopene concentrations are associated with a decreased risk of CVD and prostate cancer ${ }^{(10-13)}$.

Lycopene has eleven conjugated double bonds and each of them could be either in an $(E)$ or $(Z)$ configuration. (All-E) lycopene is the predominant isomer in plants, representing about $80-97 \%$ of total lycopene in tomatoes and related products $^{(14)}$. In human body fluids and tissues such as plasma, prostate, testis and skin, $25-70 \%$ of lycopene is found in various $(Z)$ forms ${ }^{(15-20)}$. The high concentrations of $(Z)$-isomers in vivo trigger the hypothesis that they are more bioavailable and/or that (all-E) lycopene is transformed into $(Z)$-isomers within the human body. Moreover, when a tomato-based meal rich in the (all-E) isomer is consumed daily over a few weeks, plasma lycopene concentration increases and its main part (60\%) is present as $(Z)$-isomers ${ }^{(21)}$. Recently, we have demonstrated that lycopene isomerisation takes place in the enterocytes and not in the gastrointestinal lumen ${ }^{(22)}$.

In terms of bioactivity, Shi \& Le Maguer ${ }^{(14)}$ indicated that the biological potency of ( $Z$ )-lycopene isomers is different from that of the (all-E) form. Böhm et al. ${ }^{(23)}$ reported that some $(Z)$-isomers have a stronger in vitro antioxidant activity than the (all- $E$ ) form. For these reasons, $(Z)$-lycopene isomers are

Abbreviation: TRL, TAG-rich lipoprotein.

*Corresponding author: M. Richelle, fax +41 2178585 44, email myriam.richelle@rdls.nestle.com 
regarded as offering potentially better health benefits than the (all- $E$ )-isomer.

In the present study, we investigated, in human subjects, the effect of meals enriched in lycopene on the lycopene isomer profile of plasma TAG-rich lipoprotein (TRL) fractions. The main objective was to compare the profile of lycopene isomers appearing in TRL following the ingestion of tomato preparations rich in $(Z)$-lycopene isomers. Three different tomato preparations rich in $Z$-lycopene (about $70 \%$ of total lycopene) were produced. One preparation was rich in $5-(Z)(65 \%)$, one in $13-(Z)(42 \%)$ and one in a mixture of $9-(Z)(31 \%)$ and 13-(Z) (24\%) isomers. Another objective was to determine if the tomato preparation can affect the intestinal isomerisation of (all- $E$ ) lycopene during absorption.

\section{Experimental methods \\ Lycopene preparations}

\section{Tomato preparations rich in (Z)-lycopene isomers}

Tomato preparation rich in 5-(Z) lycopene isomer. Produced by mixing $10 \mathrm{~g}$ of tomato oleoresin in $500 \mathrm{ml}$ dichloromethane with $580 \mu \mathrm{l}$ iodine solution $(5.6 \mathrm{mg}$ iodine in $596 \mu \mathrm{l}$ methylene chloride). The mixture was photoisomerised for $10 \mathrm{~h}$ at room temperature. Photoisomerised tomato oleoresin was further enriched in 5-(Z) lycopene by successive fractionation in ethanol and in ethyl acetate. The solid fraction was then re-suspended in ethyl acetate (1:80 $\mathrm{w} / \mathrm{w})$ and the suspension centrifuged at $16900 \mathrm{~g}$ for $5 \mathrm{~min}$ at room temperature. The solid fraction was discarded and the tomato preparation enriched in 5-(Z) lycopene was obtained by distilling ethyl acetate under reduced pressure at $30^{\circ} \mathrm{C}$. This preparation provided $65 \%$ of total lycopene as 5 - $(Z)$ isomer (Table 1 ).

Tomato preparation rich in 13-(Z) lycopene isomer. A suspension of tomato oleoresin in ethyl acetate $(1: 10 \mathrm{v} / \mathrm{v})$ was refluxed for $1 \mathrm{~h}$ under stirring. After cooling to room temperature, the precipitate (approximately $10 \% \mathrm{w} / \mathrm{w}$ of initial raw material) was filtered off. This precipitate contained mainly (all-E) lycopene isomer. The isomerised tomato preparation was obtained by evaporating ethyl acetate from the filtrate under reduced pressure at $30^{\circ} \mathrm{C}$ and by azeotropic distillation after addition of water and ethanol (water:ethanol ratio: 8:2). This preparation provided $70 \%$ of total lycopene as (Z)-isomers with $42 \%$ as $13-(Z)$ lycopene (Table 1 ).

Table 1. Lycopene isomer profile of the six lycopene preparations (Percentage of the total lycopene)

\begin{tabular}{lccccc}
\hline Lycopene preparation & $x-(Z)$ & $5-(Z)$ & $9-(Z)$ & $13-(Z)$ & $($ All- $E)$ \\
\hline Tomato paste & ND & $4 \cdot 1$ & ND & $0 \cdot 1$ & $95 \cdot 8$ \\
Tomato oleoresin & ND & $4 \cdot 1$ & ND & $0 \cdot 1$ & $95 \cdot 8$ \\
Lactolycopene & ND & $4 \cdot 1$ & ND & $0 \cdot 1$ & $95 \cdot 8$ \\
Tomato preparation rich & & & & & \\
5-(Z) lycopene & ND & $65 \cdot 3$ & 1.3 & ND & $33 \cdot 4$ \\
13-(Z) lycopene & $12 \cdot 0$ & $7 \cdot 6$ & $9 \cdot 6$ & $41 \cdot 5$ & $29 \cdot 3$ \\
9- and 13-(Z) lycopene & $10 \cdot 2$ & $7 \cdot 7$ & $30 \cdot 8$ & 23.5 & $27 \cdot 8$ \\
\hline
\end{tabular}

ND, not defined.
Tomato preparation rich in 9- and 13-(Z) lycopene isomers. A similar process as the one described for the tomato preparation rich in $13-(Z)$ lycopene was performed, with the exception that the heating duration had been increased up to $24 \mathrm{~h}$. This preparation provided $72 \%$ of total lycopene as $(Z)$-isomers, with $31 \%$ as $9-(Z)$ lycopene and $24 \%$ as $13-(Z)$ lycopene (Table 1).

Tomato preparations rich in (all-E) lycopene. Three tomato products, i.e. a commercial tomato paste packed in a $300 \mathrm{~g}$ tube (Thomy, Vevey, Switzerland), a tomato oleoresin (Indena, Milan, Italy) and a Lactolycopene ${ }^{\mathrm{TM}}$ preparation (Indena), contained about $95 \%$ of total lycopene as the (all-E) isomer.

The lycopene isomer profiles of the tomato supplements are presented in Fig. 1 and Table 1. With the exception of tomato paste, the daily doses of test products were packed separately as aliquots in light-protected containers, sealed under $\mathrm{N}_{2}$ and stored at $-80^{\circ} \mathrm{C}$ during the course of the study. The lycopene content and profile of isomers were shown to be stable for 6 months at $-40^{\circ} \mathrm{C}$ in the tomato products, indicating that the storage conditions were adequate.

\section{Subjects}

A total of thirty healthy men were enrolled in the study. Of these, twenty-seven subjects, aged 24 (SEM 1) years, completed the study. Their mean starting body weight was 70 (SEM 1) kg and BMI 22.5 (SEM 0.3 ) kg/m². Subjects were normolipidaemic, i.e. they had a ratio of plasma cholesterol to HDL cholesterol $<5.0$ and plasma TAG concentrations $<1.5 \mathrm{mmol} / \mathrm{l}$. Because of the large amount of blood that was drawn during the study, subjects were required to have a blood $\mathrm{Hb}$ concentration $>1.3 \mathrm{~g} / \mathrm{l}$ as inclusion criteria. The subjects did not use medication; neither did they have a history of gastrointestinal disease or lipid metabolic disorders. Three volunteers abandoned the trial before the end for the following reasons: unavailability, medical treatment related to an eye injury, nausea related to the consumption of fatty meals.

The present study was conducted according to the guidelines laid down in the Declaration of Helsinki and all procedures involving human subjects were approved by the ethical committee of Marseille (Marseille, France). All subjects received information on background and design of the study and gave written informed consent before participation. They were free to withdraw from the study at any time.

\section{Study design}

The study was a randomised, six-period, six-treatment crossover clinical trial with a washout period of 3 weeks minimum. Subjects and technicians were blinded while the investigator was blinded for five tomato products but not for the tomato paste which was a commercial product. Subjects were asked to refrain for $48 \mathrm{~h}$ before the postprandial tests from eating lycopene-rich products: tomatoes (crude, cooked and tomato sauces including ketchup and harissa), pizza, ratatouille, lasagna, pasta including tomato sauce, watermelon, pink grapefruits and guava. In addition to this dietary 
restriction, the subjects ate a standard meal the evening before the postprandial tests consisting of green vegetables, a source of cereals (pasta, bread or rice), lean meat or fish, low-fat natural yoghurt, one fruit and mineral water. They had to consume this dinner in the evening between 19.00 and 20.00 hours. These recommendations were checked by the investigator on the postprandial test day.

After an overnight fast, subjects arrived at the Clinical Pharmacology and Therapeutic Trial Center of the University of Marseille (Marseille, France) and consumed a standard meal consisting of semolina $(70 \mathrm{~g})$ cooked in $200 \mathrm{ml}$ hot water, white bread ( $40 \mathrm{~g})$, egg-whites $(60 \mathrm{~g})$, groundnut oil ( $40 \mathrm{~g})$, natural yoghurt $(125 \mathrm{~g})$, sugar $(5 \mathrm{~g})$ and water $(330 \mathrm{~g})$. This standard meal provided $842 \mathrm{kcal}(3520 \mathrm{~kJ})$ with the following nutrient composition: protein (11.7\%), carbohydrates (39.3\%) and lipids (49.0\%). A portion of one of the six lycopene preparations adapted to provide $25 \mathrm{mg}$ of lycopene was incorporated into the test meal just before its consumption. This meal was consumed within $30 \mathrm{~min}$. No other food was allowed over the following $6 \mathrm{~h}$, but the subjects were allowed to drink bottled water up to a maximum of $330 \mathrm{ml}$. Fasting blood was drawn from an anticubital vein by venepuncture into an evacuated tube containing potassium EDTA $/ K_{3}$ that was immediately placed in an ice-water-bath and covered with an aluminium foil to avoid light exposure. Blood samples were collected $20 \mathrm{~min}$ and $5 \mathrm{~min}$ before consumption of the standard meal as well as after 2, 3, 4, 5 and $6 \mathrm{~h}$ post-absorption.

This short postprandial time frame, i.e. $6 \mathrm{~h}$, was chosen to assess lycopene isomerisation within the intestine with as low contamination by lycopene isomers coming from other organs (mainly the liver) as possible. Indeed, during the postprandial period, chylomicrons secreted by the intestine, which contained lycopene coming from the meal, are transformed into chylomicron remnants following TAG hydrolysis by lipoprotein lipase. These chylomicron remnants are taken up by the liver. A fraction of dietary lycopene is then incorporated in VLDL and released in the blood. It is not known, but possible, that an isomerisation of lycopene also occurs in the liver leading to an increase in blood concentration of lycopene isomers. Because liver VLDL start to be produced some hours after a fat meal intake, the longer the postprandial period, the higher the level in the blood of VLDL coming from the liver. As, technically, it is quite complicated to isolate the chylomicron remnants from the VLDL, we decided to collect the TRL during the initial postprandial period so that they contain mostly lipoproteins of intestinal origin.

\section{Blood sample preparation}

Tubes containing the blood were protected from light, stored at $4^{\circ} \mathrm{C}$ and then centrifuged within $2 \mathrm{~h}\left(10 \mathrm{~min}, 4^{\circ} \mathrm{C}, 878 \mathrm{~g}\right)$ to separate the plasma. On the test day, plasma $(6 \mathrm{ml})$ was overlaid with $0.9 \% \mathrm{NaCl}$ solution and centrifuged for $28 \mathrm{~min}$ at $130000 \mathrm{~g}$ at $10^{\circ} \mathrm{C}$ in an SW41Ti rotor (Beckman, Fullerton, CA, USA) in an L7 ultracentrifuge (Beckman). The upper phase containing TRL, i.e. mainly chylomicrons and chylomicron remnants, was collected. Immediately after recovery,
TRL were divided into aliquots and immediately stored at $-80^{\circ} \mathrm{C}$ before lycopene isomer profile determination.

\section{Analytical procedures}

The profile of lycopene isomers was determined according to the method described previously by Schierle et $a l{ }^{(18)}$ for lycopene products (Fig. 1), and according to the method of Ferruzzi et al. ${ }^{(24)}$ for TRL. The main lycopene isomers identified were 5-( $Z$ ), 9-( $Z$ ), 13-( $Z$ ) and (all- $E$ ) lycopene. Minor compounds eluting between 24 and $48 \mathrm{~min}$ (Fig. 1) were identified as ( $Z$ )-lycopene by LC-MS/MS using an Applied Biosystems APCI 4000 LC-MS/MS (Foster City, CA, USA). The following conditions were used: isocratic flow, $1 \mathrm{ml} / \mathrm{min}$; declustering potential, $130 \mathrm{~V}$; 60 pounds per square inch $(414 \mathrm{kPa}) \mathrm{N}_{2}$; capillary voltage, $22 \mathrm{~V}$; vaporiser temperature, $400^{\circ} \mathrm{C}$; corona needle, $5 \mathrm{~mA}$; the fragmentation conditions used were those described by dos Anjos Ferreira et $a l .{ }^{(25)}$. The peak areas of unidentified $(Z)$-lycopene isomers were summed up and reported as $x$-(Z) lycopene (Table 1 ).

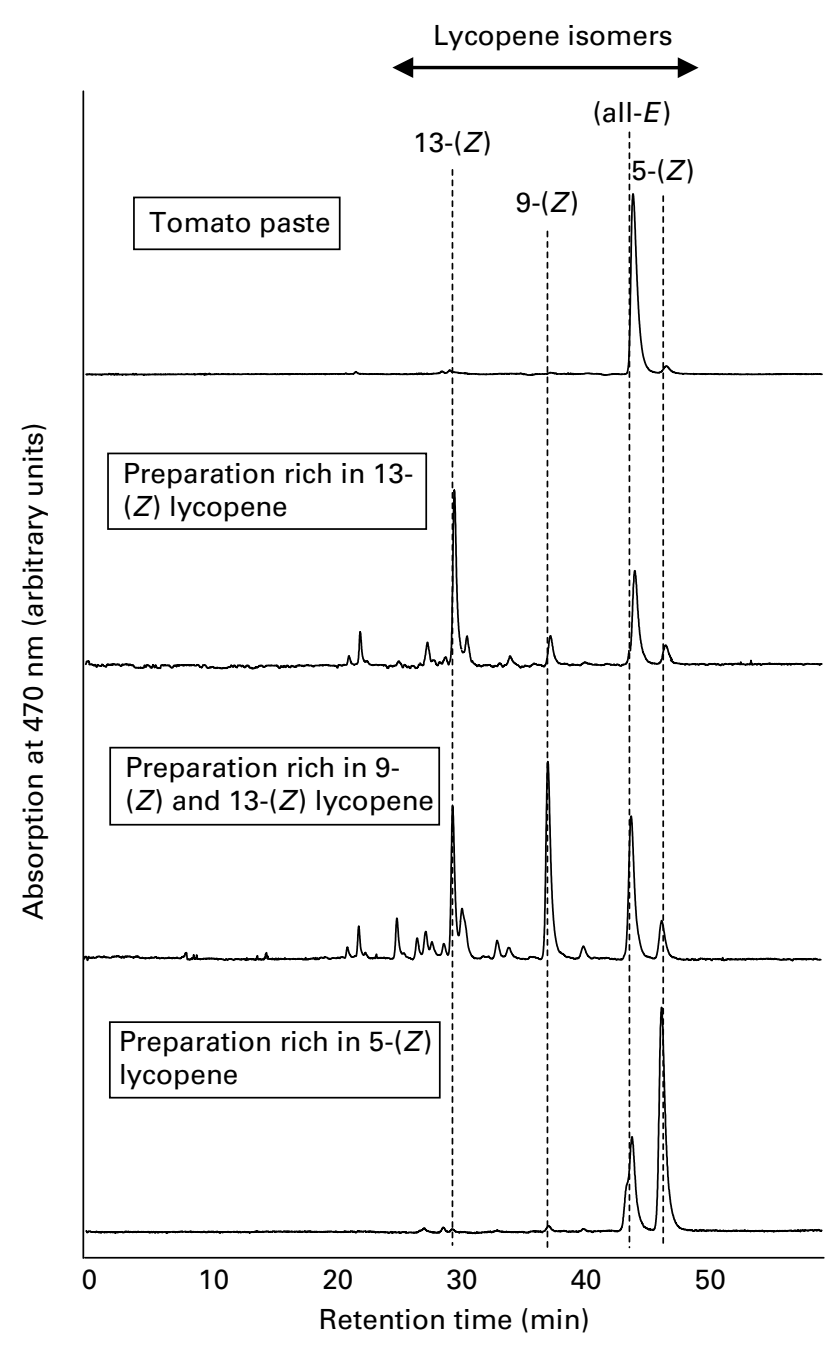

Fig. 1. HPLC chromatograms of the lycopene isomer profiles in the tomato paste rich in (all-E) lycopene and in the three tomato preparations rich in (Z)-lycopene isomers. 


\section{Data analyses}

The raw data consisted of TRL-lycopene isomer concentrations (expressed in $\mathrm{mmol} / \mathrm{l}$ ) of five lycopene isomers, i.e. $x$ - $(Z)$, 5-(Z), 9-(Z), 13-(Z) and (all-E), measured at six time points (before and, 2, 3, 4, 5 and $6 \mathrm{~h}$ after ingestion of the standardised meal containing lycopene) for each of the six different test meals and each of the twenty-seven subjects. These raw data were averaged for the twenty-seven subjects and presented as means with their standard errors for each time point (Fig. 2). The baseline was the average of the two lycopene concentrations measured in plasma samples collected
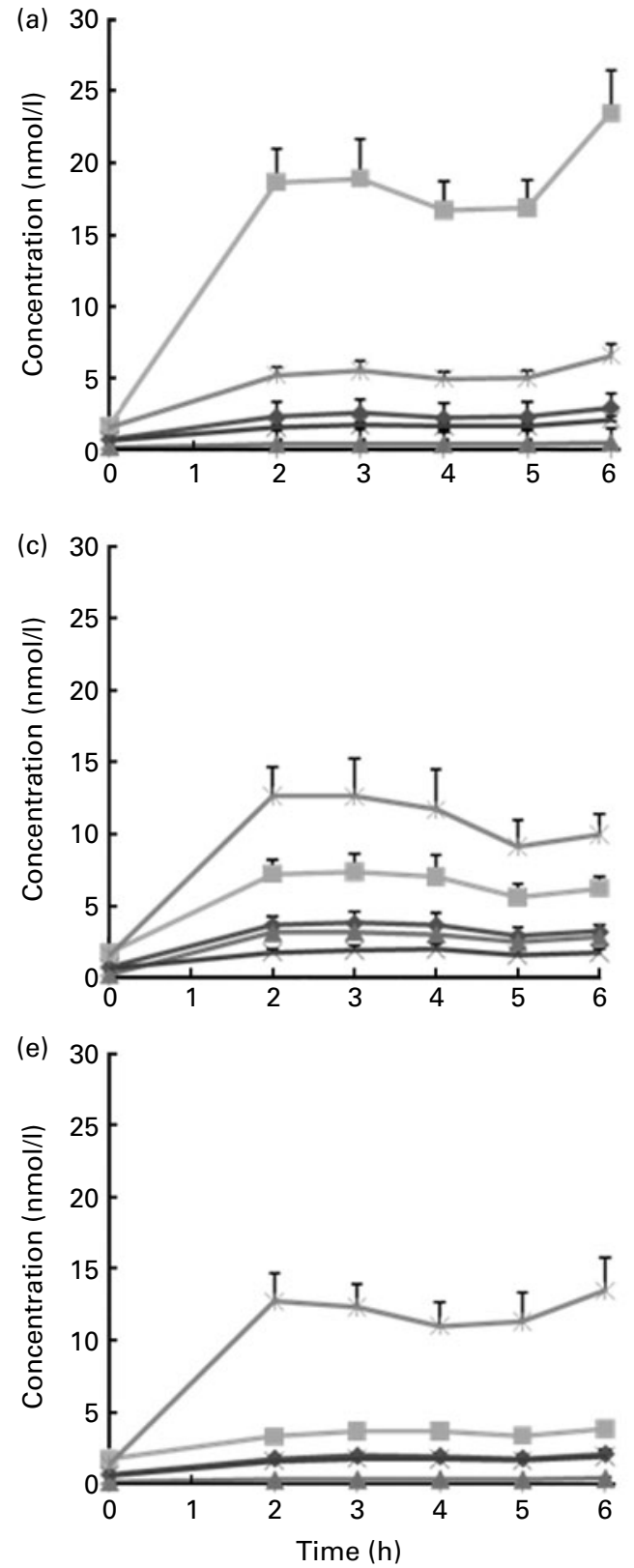

before consumption of the meal. As our goal was to determine lycopene isomerisation during the absorption phase, we voluntarily limited the measurement of lycopene isomer profiles to a short period of time after lycopene intake. Thus, we measured mainly lycopene present in chylomicrons in the TRL fraction, with only some lycopene present in the VLDL fraction coming from the liver. These purely descriptive analyses confirmed that lycopene concentrations did not return to baseline $6 \mathrm{~h}$ after ingestion of the test meal. In consequence, no formal kinetics modelling (e.g. area under the curve, $T_{\max }$ or $C_{\max }$ ) was further considered. In order to complete this descriptive data analysis and to simplify the

(b)

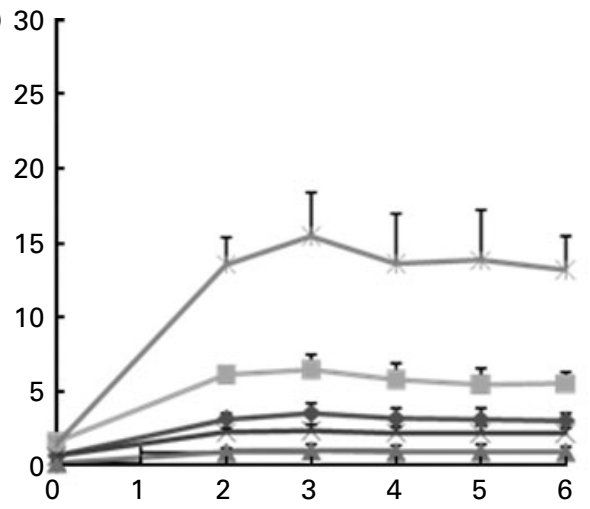

(d)

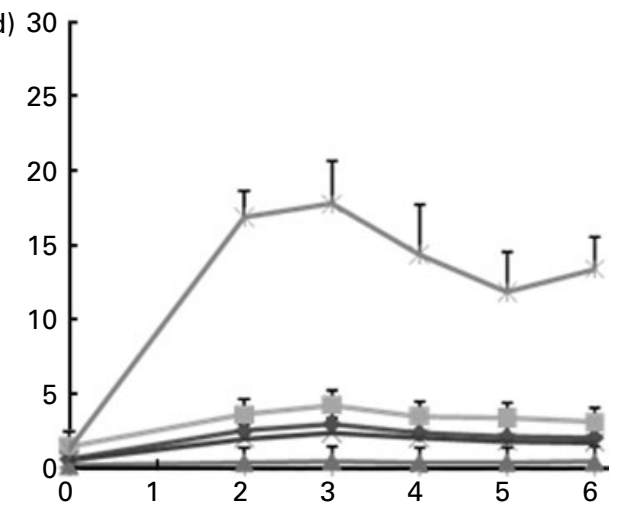

(f)

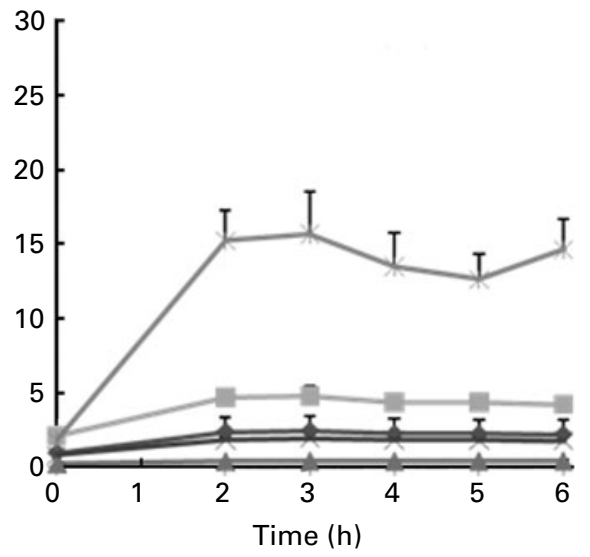

Fig. 2. TAG-rich lipoproteins-lycopene isomer concentrations over $6 \mathrm{~h}$ post-consumption of the standard meals providing $25 \mathrm{mg}$ lycopene from various sources. (a) Preparation rich in 5-(Z) lycopene, (b) preparation rich in 13-(Z) lycopene, (c) preparation rich in 9-(Z) and 13-(Z) lycopene, (d) tomato paste rich in (all- $E$ ) lycopene, (e) lactolycopene rich in (all-E) lycopene and (f) oleoresin rich in (all- $E$ ) lycopene. Values are means, with their standard errors represented by vertical bars (nmol/l, $n$ 27). $x$-(Z) lycopene $(\bullet) ; 5-(Z)$ lycopene $(\mathbf{\square}) ; 9-(Z)$ lycopene $(\mathbf{\Lambda}) ; 13-(Z)$ lycopene $(X)$; (all- $E$ ) lycopene $\left(^{*}\right)$. 
discussion of results, individual lycopene isomers were expressed as relative percentage of the total lycopene calculated as the sum of the five lycopene isomers, i.e. $x$ - $(Z), 5$ (Z), 9-(Z), 13-(Z) and (all-E) (Fig. 3).

\section{Results}

Following the ingestion of the six standardised meals supplemented with lycopene, TRL-lycopene concentrations increased promptly, reaching a first maximum concentration between 2 and $4 \mathrm{~h}$, and started to increase again after $5 \mathrm{~h}$ (Fig. 2). Since lycopene isomer profiles were determined
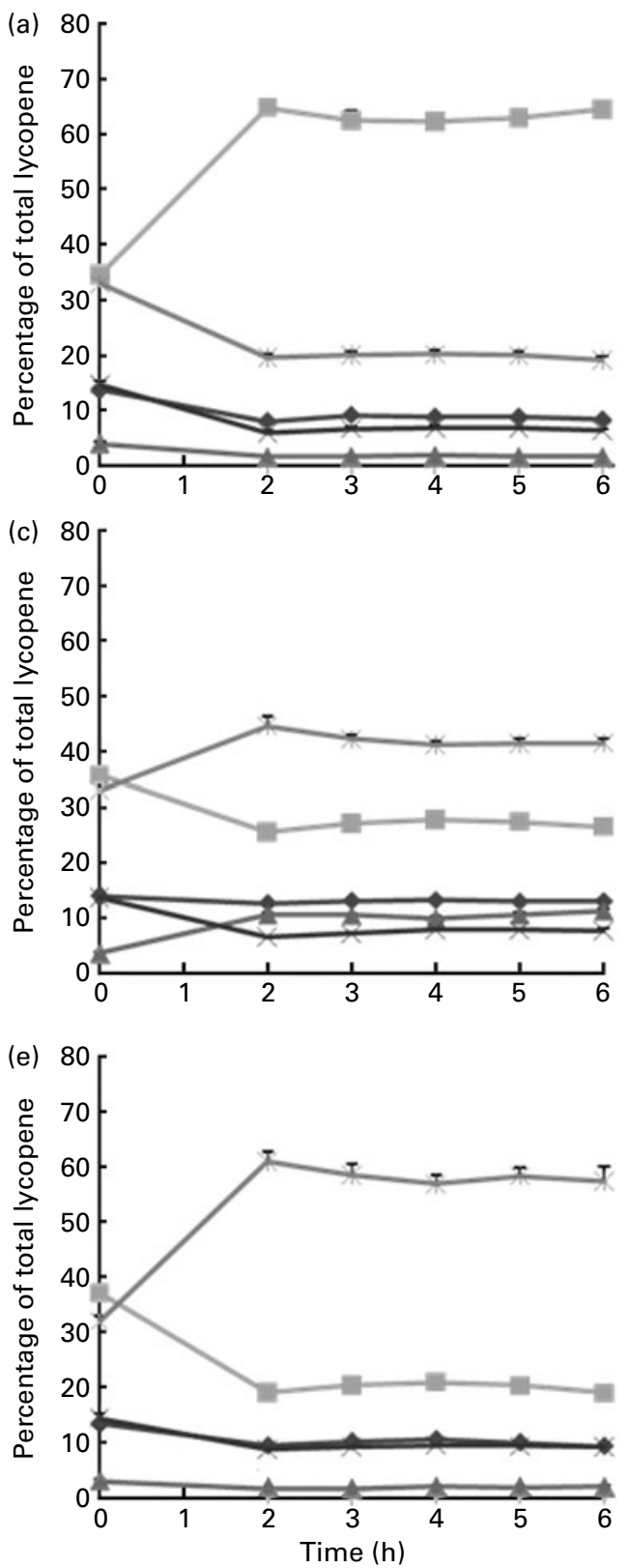

over a short period of time only, the second maximum was not well characterised. These two rises of lycopene concentrations are in agreement with the known lycopene absorption processes and show a first appearance of dietary lycopene in chylomicrons (before $4 \mathrm{~h}$ ) and a subsequent incorporation of dietary lycopene chylomicrons into newly released VLDL from the liver (after $5 \mathrm{~h}$ ). In consequence, our choice for a short postprandial time frame $(0-6 \mathrm{~h})$, which accounts for the appearance of lycopene in chylomicrons, is ideal to determine lycopene isomerisation within the intestine.

Interestingly, the profile of lycopene isomers expressed in percentage (Fig. 3) did not vary over the $6 \mathrm{~h}$ post-absorption.
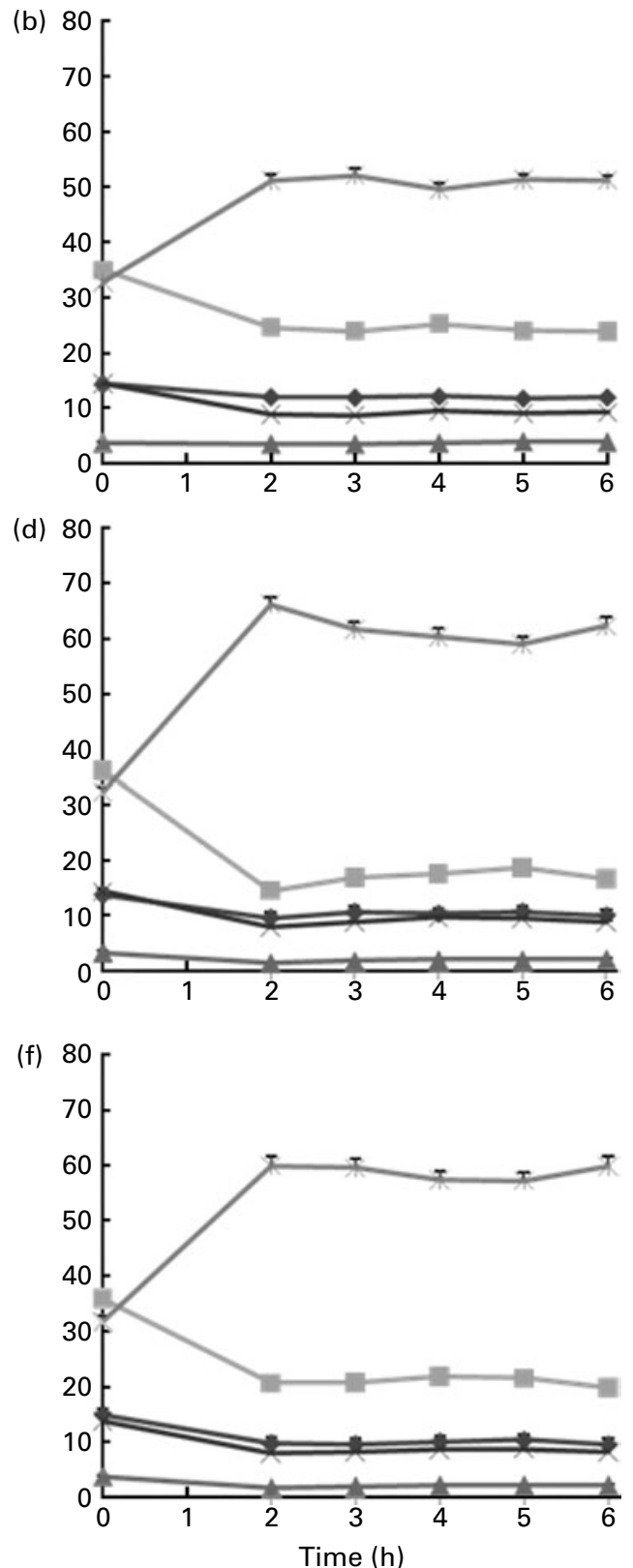

Fig. 3. TAG-rich lipoproteins-lycopene isomer profiles over $6 \mathrm{~h}$ post-consumption of the standard meals providing $25 \mathrm{mg}$ lycopene from various sources. (a) Preparation rich in 5-(Z) lycopene, (b) preparation rich in 13-(Z) lycopene, (c) preparation rich in 9-( $Z$ ) and 13-( $Z$ ) lycopene, (d) tomato paste rich in (all- $E$ ) lycopene, (e) lactolycopene rich in (all- $E$ ) lycopene and (f) oleoresin rich in (all- $E$ ) lycopene. Values are means, with their standard errors expressed as percentage of the total lycopene represented by vertical bars $(n 27) . x-(Z)$ lycopene $(\bullet)$; 5- $(Z)$ lycopene $(\boldsymbol{\square})$; 9- $(Z)$ lycopene $(\boldsymbol{\Lambda})$; $13-(Z)$ lycopene $(X)$; (all- $E)$ lycopene $\left({ }^{\star}\right)$. 
Moreover, the inter-individual variability of concentrations (Fig. 2) and percentages of lycopene (Fig. 3) were very small.

\section{Proportion of lycopene isomers in TAG-rich lipoprotein after consumption of tomato preparations rich in (Z)-lycopene}

Consumption of a tomato preparation rich $(65 \%)$ in $5-(Z)$ lycopene resulted in a close proportion $(60 \%)$ of this isomer in TRL, suggesting a good absorption and a low isomerisation of this isomer in the enterocytes (Figs. 2(a) and 3(a)). The other lycopene isomers observed in TRL consisted of $20 \%$ of (all- $E$ ), $9 \%$ of $x-(Z), 7 \%$ of $13-(Z)$ and $2 \%$ of $9-(Z)$. Since these $(Z)$-isomers were not present in the diet, their appearance in TRL suggests that they were formed during absorption by isomerisation of the (all-E) lycopene.

Only a low amount (9\%) of the 13-(Z) isomer was measured in TRL following the intake of a tomato preparation which contained $42 \%$ of this isomer (Figs. 2(b) and 3(b)). Other lycopene isomers measured in TRL were mainly the (all-E) (about $50 \%)$ and the $5-(Z)(25 \%)$ isomers, suggesting that part of the 13-(Z) lycopene was isomerised into these isomers during absorption

Eating the tomato preparation rich in a mixture of 9-( $Z)$ and 13-( $Z$ ) lycopene induced a release of lycopene in TRL containing mostly the (all-E) (42\%) and 5-(Z) (27\%) isomers (Figs. $2(\mathrm{c})$ and 3(c)), suggesting that the 9-( $Z$ ) and $13-(Z)$ isomers were isomerised into the (all- $E$ ) and $5-(Z)$ isomers.

\section{Proportion of lycopene isomers in TAG-rich lipoprotein after consumption of tomato preparations rich in (all-E) lycopene}

Consumption of the tomato paste rich in (all-E) lycopene (about 95\%) resulted in observing various lycopene isomers in TRL, i.e. roughly $60 \%$ (all-E) and $40 \%(Z$ )-isomers, with about $18 \%$ of $5-(Z), 10 \%$ of $13-(Z)$ and $x-(Z)$, and $2 \%$ of 9-( $Z$ ) (Figs. 2(d) and 3(d)). The appearance of isomers that were not detected in the tomato paste, i.e. 9-(Z), 13-(Z) and $x-(Z)$, strongly suggests that part of the (all-E) was isomerised during absorption.

The same profile of lycopene isomers in TRL was also observed with the two other tomato preparations, i.e. lactolycopene and oleoresin, containing about 95\% (all-E) lycopene (Figs. 2(e), (f) and 3(e), (f)). These results indicate that the three tomato preparations rich in the (all-E) isomer were similarly isomerised during their absorption in human subjects and, therefore, that the lycopene food matrix did not influence it.

\section{Discussion}

We have recently demonstrated that, during absorption, a significant isomerisation of (all- $E$ ) lycopene into various $(Z)$-lycopene isomers takes place in human enterocytes ${ }^{(22)}$. The first objective of the present study was to determine if the lycopene isomer profile in the blood circulation was affected by the isomer profile in the meal.
Consumption of the tomato preparation rich in 5 - $(Z)$ lycopene led to a very high proportion of 5-(Z) lycopene isomer in TRL (about $60 \%$ ), which indicates either a good absorption, or a greater thermodynamical stability ${ }^{(26)}$ of this isomer, or both.

The consumption of either a preparation rich in a mixture of 9-( $Z)$ and $13-(Z)$ or a preparation rich in $13-(Z)$ lycopene was associated with the release of TRL which contained lower proportions of these two isomers as compared to those present in the tomato preparations. These results indicate either a low absorption of these isomers or an isomerisation of these isomers into more thermodynamically stable lycopene isomers, i.e. (all- $E$ ) and 5-(Z), during absorption. The second hypothesis seems the most likely, as the proportion of the (all-E) and 5-(Z) isomers measured in TRL after intake of the preparation rich in 9- $(Z)$ and $13-(Z)$ isomers was higher than in the preparation itself.

These results show that although the human intestine is able to modify the profile of dietary lycopene isomers which is finally found in the body, the resulting profile is not the same for all isomer sources supplied but is dependent on the initial relative proportions of these isomers existing in the meal. This suggests that the mechanism of $(E)$-to- $(Z)$ conversion can be overwhelmed by offering high amounts of dietary lycopene $(Z)$-isomers, e.g. 5-(Z). It is, therefore, possible to modulate the profile of $(Z)$-isomers which enter the human body via the intestinal lipoproteins by a lycopene isomer-rich diet.

Besides, it is well known that the bioavailability of (all- $E$ ) lycopene is dependent on the tomato preparation ${ }^{(21)}$. In agreement with results reported in the literature ${ }^{(15,17,27)}$, we have shown that consuming a tomato paste which contained mainly (95\%) (all-E) lycopene resulted in secretion of TRL containing $40 \%$ of lycopene $(Z)$-isomers ${ }^{(21,22)}$, which suggests that part of the ingested (all-E) lycopene was converted into $(Z)$-isomers. The secondary objective of this work was to assess whether the preparation of (all-E) lycopene affects the isomerisation of the (all- $E$ ) lycopene during intestinal absorption. Present results show that consumption of the three tomato preparations rich in the (all-E) isomer (tomato paste, (all-E) oleoresin and lactolycopene) resulted in a similar isomerisation of the (all-E) lycopene. This shows that lycopene isomerisation occurring in the enterocytes is not significantly affected by the lycopene preparation. Another key observation is that the main ( $Z$ )-isomer recovered in TRL was the 5-(Z) lycopene (about $20 \%$ of the total lycopene content). This suggests that either the $5-(Z)$ lycopene is specifically formed during isomerisation of the (all- $E$ ) lycopene, or the 5-( $Z$ ) isomer, being more stable than the others ${ }^{(26)}$, leads to its preferential accumulation during absorption, or both.

Interestingly, there is a low inter-individual variation of lycopene isomerisation during intestinal absorption, which is in agreement with previous results observed with tomato paste $^{(22)}$.

( $Z$ )-lycopene isomers have been discussed for their specific bioactivity. Indeed, individual $(Z)$-lycopene isomers exhibit different antioxidant activities ${ }^{(23)}$ and some human organs like prostate ${ }^{(16)}$ and skin accumulate high amounts of 
(Z)-lycopene isomers. More research is needed to evaluate how chronic supplementation with a diet enriched in ( $Z$ )-lycopene isomers will affect their distribution in tissues and subsequently will modulate lycopene bioactivity in humans.

\section{Acknowledgements}

The present study was funded in full by the Nestlé Research Center, Lausanne, Switzerland. M. R., P. L., C. J., P. B. and K. B. conceived and designed the study. I. T., P. L., A.-F. M. and $\mathrm{K}$. B. achieved the design and production of formulations of the lycopene products. C. J. coordinated the trial and supervised the analytical aspects. C. J. and P. B. contributed to the development of analytical methods, lycopene analysis and data collection. M. R., P. L., C. J., P. B., K. B. and A. R. analysed the data. M. R. wrote the manuscript, and all authors were involved in interpreting the results and in critical revision of the paper. No author has any advisory board affiliation. The authors declare that they have no conflicts of interest.

\section{References}

1. Gann PH, Ma J, Giovanucci E, et al. (1999) Lower prostate cancer risk in men with elevated plasma lycopene levels: results of a prospective analysis. Cancer Res 59, 1225-1230.

2. Blaner WS \& Olson JA (1994) Retinol and retinoic acid metabolism. In The Retinoids: Biology, Chemistry and Medicine, 2nd ed., pp. 229-255 [MB Sporin, AB Roberts and S Goodman, editors]. New York, NY: Raven Press.

3. Chalabi N, Delort L, Le Corre L, et al. (2006) Gene signature of breast cancer cell lines treated with lycopene. Pharmacogenomics 7, 663-672.

4. Chalabi N, Le Corre L, Maurizis JC, et al. (2004) The effect of lycopene on the proliferation of human breast cells and BRCA1and BRCA2 gene expression. Eur J Cancer 40, 1769-1775.

5. Chalabi N, Satih S, Delort L, et al. (2007) Expression profiling by whole genome microarray hybridization reveals differential gene expression in breast cancer cell lines after lycopene exposure. Biochim Biophys Acta 1796, 124-130.

6. Huang CS, Shih MK, Chuang $\mathrm{CH}$, et al. (2005) Lycopene inhibits cell migration and invasion and upregulates Nm23-H1 in a highly invasive hepatocarcinoma, SH-Hep-1 cells. J Nutr 135, 2119-2123.

7. Nahum A, Zeller L, Danilenko M, et al. (2006) Lycopene inhibition of IGF-induced cancer cell growth depends on the level of cyclin D1. Eur J Nutr 45, 275-282.

8. Liu C, Russell RM \& Wang XD (2006) Lycopene supplementation prevents smoke induced changes in p53 phosphorylation, cell proliferation, and apoptosis in the gastric mucosa of ferrets. J Nut 136, 106-111.

9. Russell RM (1998) Physiological and clinical significance of carotenoids. Int J Vitam Nutr Res 68, 349-353.

10. Rao AV (2002) Lycopene, tomatoes, and the prevention of coronary heart disease. Exp Biol Med (Maywood) 227, 908-913.
11. Levy J, Bosin E, Feldman B, et al. (1995) Lycopene is a more potent inhibitor of human cancer cell proliferation than either $\alpha$-carotene or $\beta$-carotene. Nutr Cancer 24, 257-266.

12. Giovannucci E, Ascherio A, Rimm EB, et al. (1995) Intake of carotenoids and retinol in relation to risk of prostate cancer. J Natl Cancer Inst 87, 1767-1776.

13. Etminan M, Takkouche B \& Caamaño-Isorna F (2004) The role of tomato products and lycopene in the prevention of prostate cancer: a meta-analysis of observational studies. Cancer Epidemiol Biomark Prevent 13, 340-345.

14. Shi J \& Le Maguer M (2000) Lycopene in tomatoes: chemical and physical properties affected by food processing. Crit Rev Food Sci Nutr 40, 1-42.

15. Alien CM, Smith AM, Clinton SK, et al. (2002) Tomato consumption increases lycopene isomer concentrations in breast milk and plasma of lactating women. J Am Diet Assoc 102, 1257-1262.

16. Clinton SK, Emenhiser C, Schwartz SJ, et al. (1996) Cis-trans lycopene isomers, carotenoids and retinol in the human prostate. Cancer Epidemiol Biomarkers Prev 5, 823-833.

17. Holloway DE, Yang M, Paganga G, et al. (2000) Isomerization of dietary lycopene during assimilation and transport in plasma. Free Radic Res 32, 93-102.

18. Schierle J, Bretzel W, Bühler I, et al. (1997) Content and isomeric ratio of lycopene in food and human plasma. Food Chem 59, 459-465.

19. Stahl W \& Sies H (1992) Uptake of lycopene and its geometrical isomers is greater from heat-processed than from unprocessed tomato juice in humans. J Nutr 122, 2161-2166.

20. Wu K, Schwartz SJ, Platz EA, et al. (2003) Variations in plasma lycopene and specific isomers over time in a cohort of US men. J Nutr 133, 1930-1936.

21. Richelle M, Bortlik K, Liardet S, et al. (2002) A food-based formulation provides lycopene with the same bioavailability to humans as that from tomato paste. J Nutr 132, 404-408.

22. Richelle M, Sanchez B, Tavazzi I, et al. (2010) Lycopene isomerisation takes place within enterocytes during absorption in human subjects. Br J Nutr 103, 1800-1807.

23. Böhm V, Puspitasari-Nienaber NL, Ferruzzi MG, et al. (2002) Trolox equivalent antioxidant capacity of different geometrical isomers of $\alpha$-carotene, $\beta$-carotene, lycopene, and zeaxanthin. J Agric Food Chem 50, 221-226.

24. Ferruzzi MG, Nguyen ML, Sander LC, et al. (2001) Analysis of lycopene geometrical isomers in biological microsamples by liquid chromatography with coulometric array detection. J Chromatogr B Biomed Sci Appl 760, 289-299.

25. dos Anjos Ferreira AL, Yeum K-J, Russell RM, et al. (2004) Enzymatic and oxidative metabolites of lycopene. $J$ Nutr Biochem 15, 493-502.

26. Chasse GA, Mak ML, Deretey E, et al. (2001) An ab initio computational study on selected lycopene isomers. $J \mathrm{Mol}$ Struc: Theochem 571, 27-37.

27. Fröhlich K, Kaufmann K, Bitsch R, et al. (2006) Effects of ingestion of tomatoes, tomato juice and tomato purée on contents of lycopene isomers, tocopherols and ascorbic acid in human plasma as well as on lycopene isomer pattern. Br J Nutr 95, 734-741. 